Trauma Berufskrankh 2012 · 14[Suppl 3]:352-357 DOI 10.1007/s10039-011-1823-z

Online publiziert: 22. März 2012

(c) Springer-Verlag 2012
U. Kneser · A. Arkudas · J.P. Beier · A. Dragu · R.E. Horch

Plastisch- und Handchirurgische Klinik, Universitätsklinikum Erlangen

\title{
Knöcherne und ligamentäre Verletzungen des Handgelenks
}

\section{Im Kontext der schweren kombinierten Handverletzung}

Knöcherne und ligamentäre Schädigungen des Handgelenks stellen häufige Verletzungen der oberen Extremität dar, welche auch im Kontext der schweren kombinierten Handverletzung auftreten können [6,7]. Dabei kann es in Abhängigkeit vom Schädigungsmechanismus zu ausgedehnten Destruktionen der knöchernen und ligamentären Anteile des Carpus kommen. Im vorliegenden Beitrag soll eine Übersicht über die anatomischen Grundlagen, Mechanismen, Diagnostik und Therapie der karpalen Verletzungen gegeben werden.

\section{Anatomie}

Das Handgelenk, welches die mechanische Verbindung zwischen der Hand und dem Unterarm darstellt, zeichnet sich durch eine komplexe dreidimensionale Anatomie aus [19, 24]. Dabei bewegt sich die Hand in einer Vielzahl von nebenund hintereinander geschalteten Gelenken relativ gegenüber dem Unterarm.

Carpus. Er setzt sich aus einer körpernahen und einer körperfernen Knochenkette zusammen, wobei Erstere von radial aus dem Os scaphoideum, dem Os lunatum, dem Os triquetrum sowie dem Os pisiforme, welches die Funktion eines Sesambeins besitzt, besteht. Die distale Handwurzelreihe setzt sich aus dem Os trapezium, dem Os trapezoideum, dem
Os capitatum sowie dem Os hamatum zusammen.

Während der Carpus historisch als ein unverformbarer Block von fest zusammengefügten Einzelknochen gesehen wurde, besteht gemäß aktuellen anatomischen Untersuchungen kein Zweifel, dass er eine wesentlich komplexere biomechanische Konstellation besitzt. So weist v. a. die proximale Handwurzelreihe, welche zwischen das Radio- und das sog. Mediokarpalgelenk geschaltet ist, eine große Beweglichkeit auf. Es wird angenommen, dass in Neutralstellung des Handgelenks etwa 50\% der Kraft über die Fossa scaphoidea und etwa $40 \%$ über die Fossa lunata in den Radius eingeleitet werden. Während in der proximalen Handwurzelreihe v. a. Rotationsbewegungen zwischen dem Os scaphoideum und dem Os lunatum, eingeschränkt auch zwischen dem Os lunatum und dem Os triquetrum möglich sind, erlauben die interossären Bänder der distalen Handwurzelreihe nur geringere Ausschläge.

Bandsystem des Karpalgelenks. Nach Lichtman u. Martin [14] besteht das Karpalgelenk aus einem dynamischen Ringsystem, welches unter Spannung steht. Der Zusammenhalt dieses Systems wird auch durch ligamentäre Verbindungen sichergestellt. Diese lassen sich in sog. extrinsische und intrinsische Bänder unterteilen. Von großer Bedeutung für die Bio- mechanik des Carpus sind im intrinsischen Bandsystem die Bandverbindung zwischen dem Os lunatum und dem Os scaphoideum sowie die zwischen dem Os lunatum und dem Os triquetrum $[1,20$, 24]. Die skaphulonäre Bandverbindung lässt sich in einen palmarseitigen, einen proximalen und einen dorsalen Anteil unterteilen. Dabei ist Letzterer mit einer Dicke von bis zu $3 \mathrm{~mm}$ und sehr kurzen, quer verlaufenden Fasern im Hinblick auf die Stabilität von größter Bedeutung [13]. Im Gegensatz hierzu ist der palmarseitige Anteil der Bandverbindung zwischen Os lunatum und Os triquetrum von größerer biomechanischer Relevanz.

Neben den genannten Bändern existiert eine Vielzahl verschiedener Ligamente des extrinsischen bzw. intrinsischen karpalen Bandsystems, welche im Rahmen von schweren kombinierten Handverletzungen zerstört werden können [21]. Die chirurgische Rekonstruktion jeder einzelner dieser Strukturen ist jedoch im Rahmen der Erstversorgung nicht immer zwangsläufig erforderlich.

\section{Biomechanik und typische Verletzungsmuster}

Um im Alltag die nahezu uneingeschränkte Positionierung der Hand im Raum zu ermöglichen, muss das Handgelenk zum einen eine große Mobilität aufweisen, zum anderen jedoch auch die Ein- 
leitung signifikanter Kräfte in den Unterarm ermöglichen. Viele teilweise widersprüchliche funktionelle Anforderungen können nur durch eine perfekte Interaktion zwischen den knöchernen und ligamentären Anteilen sowie auch der motorischen Ansteuerung aus dem Unterarm erzielt werden.

Während der radioulnaren Bewegung des Handgelenks bewegen sich die 3 proximalen Carpalia synergistisch von einer flektierten Stellung bei Radialdeviation zu einer extendierten Stellung in der Ulnadeviation. Bereits bei physiologischen Belastungen, z. B. dem festen Greifen von Objektiven mit der Faust, wirken beträchtliche Kräfte auf das Handgelenk [11]. Es ist bekannt, dass die von allen Metacarpalia in die distale Handwurzelreihe eingeleitete Kraft um ein Vielfaches stärker ist als die an den Fingerspitzen applizierte Kraft.

\section{Verletzungsmechanismus}

Im Hinblick auf die Pathobiomechanik des Carpus kann zwischen 2 Verletzungsmechanismen unterschieden werden:

- dem direkten Verletzungsmechanismus und

- dem indirekten Verletzungsmechanismus.

Während bei Ersterem die Kraft direkt auf den betroffenen Knochen übertragen wird, wird beim indirekten Mechanismus die deformierende Kraft $u$. U. in weiter Entfernung vom betroffenen Gelenk appliziert.

Der klassische Mechanismus einer direkten Verletzung besteht z. B. in der Quetschverletzung des Carpus in einer Presse. Indirekte Verletzungen können z. B. bei extremer passiver Extension des Handgelenks, u. U. in Verbindung mit einer Ulnadeviation, auftreten. Dabei kann die Hyperextension zur Destruktion von sowohl knöchernen als auch ligamentären Strukturen führen.

\section{Verletzungsmuster}

Während bei isolierten karpalen Frakturen die Skaphoidfraktur die mit Abstand häufigste Bruchverletzung darstellt [12], kann es bei schweren kombinierten Handverletzungen in Abhängigkeit vom
Verletzungsmechanismus zur Fraktur jedes einzelnen karpalen Knochens kommen.

Karpale Luxationsfraktur. Sie stellt die klassische Folge einer indirekten Krafteinwirkung auf den Carpus dar. Nach Cooney et al. [4] können diese Verletzungen in 4 Gruppen unterteilt werden:

- „lesser arc“-Verletzung, die klassische perilunäre Luxation,

- "greater arc“-Verletzung, die transkarpale perilunäre Dislokation,

- axiale Dislokation und

- isoliert karpal-knöcherne Dislokation.

Verletzungen des großen und kleinen

Bogens. Sie werden nach Mayfield et al. $[15,16]$ in mehrere Stadien unterteilt. Diese Klassifikation beschreibt den in der Regel stattfindenden Progress der Verletzung in Folge einer axialen Belastung des extendierten und ulnardeviierten Carpus.

Im Stadium I sind das palmare radiokarpale und das skapholunäre Ligament sowie das skaphotrapeziotrapezoidale Ligament maximal angespannt. Infolgedessen kommt es entweder zur Ruptur des skaphulonären Ligaments von palmar nach dorsal oder zur Fraktur des Skaphoids.

Im Stadium II ist das Skaphoid bereits von der proximalen Handwurzelreihe entweder aufgrund eines Ligamentschadens oder einer Fraktur - weitestgehend separiert. Dies führt zur Verlagerung des Capitatums nach dorsal und letztendlich zur kompletten Dislokation der distalen Handwurzelreihe sowie des radialen Abschnitts der proximalen Handwurzelreihe nach dorsal.

Im Stadium III kommt es zur Ruptur des lunotriquetralen Ligaments oder zur Avulsionsfraktur des Os triquetrum.

Im Stadium IV tritt letztlich das Os capitatum nach proximal und verdrängt das Os lunatum mit einer Rotationsbewegung nach palmar - die perilunäre Luxation ist komplett.

Während die meisten geschlossenen perilunären Luxationen bzw. Luxationsfrakturen diesem pathomechanischen Verletzungsmuster folgen, kann es bei direkter externer Krafteinwirkung im Rahmen einer schweren kombinierten Hand- verletzung auch zu abweichenden Verletzungsmechanismen kommen.

\section{Diagnostik}

Im Folgenden soll ausschließlich die Versorgung karpaler Verletzungen dargestellt werden.

Selbstverständlich ist bei polytraumatisierten Patienten eine interdisziplinäre Initialversorgung im Schockraum erforderlich. Bei der anschließenden chirurgischen Versorgung kann u. U. die Therapie der Handverletzung von geringerer Dringlichkeit sein. Beim vital gefährdeten Patienten ist dann ggf. nur deren präliminare Versorgung möglich, während die weitergehende handchirurgische Rekonstruktion nach Stabilisierung im Intervall durchgeführt werden muss.

\section{Anamnese}

Im Rahmen der Erstversorgung sollte, falls möglich, eine kurze Anamnese bezüglich des Unfallhergangs sowie eventueller Vorschäden im Bereich der betroffenen oberen Extremität erhoben werden. Unter Umständen erlaubt eine genaue Kenntnis des Unfallmechanismus bereits eine Abschätzung des zu erwartenden Verletzungsausmaßes.

\section{Klinik}

Im Rahmen der körperlichen Untersuchung sollte das Ausmaß des Weichteiltraumas evaluiert werden. Des Weiteren lässt u. U. bereits eine Achsenfehlstellung Rückschlüsse auf karpale Verletzungen zu. Die Durchblutung der Hand muss untersucht werden, da es auch im Rahmen von geschlossenen Verletzungen zur Kompression bzw. Ruptur der Hauptgefäßachsen im Bereich des Handgelenks kommen kann. Bei wachen, kooperationsfähigen Patienten sollten des Weiteren die Sensibilität und auch die Motorik im Bereich des Daumens und der Langfinger untersucht werden.

\section{Bildgebung}

Die Röntgenuntersuchung des Handgelenks sowie des distalen Unterarmes in 2 Ebenen ist zur Planung der weiteren 
Versorgung zwingend erforderlich. In Abhängigkeit des Verletzungsmusters sollte sich auch eine Röntgenaufnahme des gesamten Unterarms sowie der Hand anschließen.

Bei Verdacht auf komplexe karpale Verletzungen ist frühzeitig die Indikation zur Computertomographie (Dünnschichttomographie des Carpus) zu stellen, da häufig nur sie eine genaue Darstellung des karpalen Verletzungsausmaßes ermöglicht ( $\bullet$ Abb. 1a,b).

Im Rahmen der Akutversorgung sind diese bildgebenden Untersuchungen in der Regel ausreichend, weder die Ultraschalldiagnostik noch die Magnetresonanztomographie sind in dieser Situation bisher als Standardverfahren etabliert. Diese Untersuchungsmodalitäten können jedoch im Rahmen der Sekundärrekonstruktion Bedeutung erlangen.

\section{Therapie}

\section{Zeitpunkt}

Komplexe karpale Verletzungen sollten in der Regel zeitnah versorgt werden.

Während geschlossene isolierte Verletzungen von Ligamenten oder Knochen, z. B. eine Zerreißung des skapholunären Bandes oder eine isolierte Skaphoidfraktur, auch frühelektiv innerhalb weniger Tage nach dem Unfallereignis adäquat therapiert werden können, sollte bereits eine geschlossene karpale Luxationsfraktur schnellstmöglich einer chirurgischen Versorgung zugeführt werden, um die Kompromittierung von Nerven und Muskeln zu vermeiden. Häufig kommt es bei nicht reponierten perilunären Luxationsfrakturen zur Einengung des N. medianus mit einem akuten Karpaltunnelsyndrom. Auch kann sich ein Kompartmentsyndrom im Bereich des Unterarms entwickeln. Offene knöcherne und ligamentäre Verletzungen des Handgelenks, z. B. auch im Rahmen von schweren kombinierten Handverletzungen, stellen einen chirurgischen Notfall dar.

Selbstverständlich gilt bei polytraumatisierten und instabilen Patienten das Prinzip „life before limb“.

Es muss berücksichtigt werden, dass eine inadäquate Erstversorgung derartiger Verletzungen zu einer prognostisch

Trauma Berufskrankh 2012 - 14[Suppl 3]:352-357 DOI 10.1007/s10039-011-1823-z

C) Springer-Verlag 2012

U. Kneser · A. Arkudas - J.P. Beier - A. Dragu - R.E. Horch

\section{Knöcherne und ligamentäre Verletzungen des Handgelenks. Im Kontext der schweren kombinierten Handverletzung}

\section{Zusammenfassung}

Knöcherne und ligamentäre Verletzungen des Handgelenks treten häufig im Rahmen einer schweren kombinierten Handverletzung auf. Die adäquate chirurgische Erstversorgung ist von hoher prognostischer Bedeutung. Es sollte daher nach Möglichkeit bereits initial eine Rekonstruktion aller verletzten funktionell relevanten Strukturen angestrebt werden. Dabei muss u. U. bereits im Rahmen der Erstversorgung die Indikation zu Teil- oder Komplettarthrodesen gestellt werden. Auch eine zeitnahe Versorgung von zusätzlich bestehenden Gefäß-, Nerven- und Sehnenverletzungen sowie von Weichteildefekten ist zwingend erforderlich. Die operative Therapie sollte möglichst eine frühfunktio- nelle Beübung zulassen. Die Versorgung derartiger komplexer karpaler Verletzungen erfolgt idealerweise in spezialisierten Zentren. Der physio- und ergotherapeutischen Nachbehandlung kommt eine große Bedeutung zu. Gegebenenfalls ist frühzeitig ein Berufshelfer hinzuzuziehen, um bei prognostisch ungünstigen Verletzungen durch Umsetzungen am Arbeitsplatz bzw. Umschulungsmaßnahmen den dauerhaft bestehenden funktionellen Einschränkungen Rechnung zu tragen.

\section{Schlüsselwörter}

Handverletzungen · Handwurzel, Verletzungen · Handgelenkverletzungen . Dislokation · Amputation

\section{Carpal fractures and injuries of carpal ligaments. In conjunction with severe combined hand injuries}

\begin{abstract}
Carpal fractures and injuries of carpal ligaments are commonly seen as consequences of severe combined hand injuries. Adequate initial surgical management is essential for optimal functional outcome. Early repair of functionally relevant carpal structures is advocated and even partial or complete wrist arthrodesis might be indicated in this phase. Also, repair of injured blood vessels, nerves and tendons as well as reconstruction of soft tissue defects that might accompany the carpal injury is mandatory. Ideally, the initial sur-
\end{abstract}

gical management allows for early physical therapy. These severe injuries should be primarily referred to specialized centres that also possess adequate facilities for physical and occupational therapy. Occupational rehabilitation after severe combined hand injuries is of great importance and requires specialized counselling.

\section{Keywords}

Hand injuries · Carpal joints, injuries . Wrist injuries · Dislocations · Amputation langfristig deutlich schlechteren Funktion führen kann.

\section{Verfahrenswahl}

Frakturen des Skaphoids sollten, falls möglich, mit einer übungsstabilen Schraubenosteosynthese (z. B. HerbertSchraube) versorgt werden.

Die osteosynthetische Behandlung von Frakturen der restlichen karpalen Knochen richtet sich nach dem Grad der Dislokation bzw. dem Verletzungsausmaß und dem Vorliegen von knöchernen Substanzdefekten. Unter Umständen sind auch hier eine Drahtosteosynthese oder nach adäquater Reposition der Verzicht auf eine weitere interne Osteosynthese angebracht. Im Falle von ligamentären Verletzungen sollten auch das skapholunare Ligament in seinem dorsalen Anteil sowie, falls betroffen, das lunotriquetrale Ligament in seinem palmarseitigen Anteil zwingend rekonstruiert werden. Dies kann in Abhängigkeit vom Verletzungsausmaß entweder mittels direkter Naht oder unter Verwendung von Knochenankern erfolgen. Gelegentlich kann auch im Rahmen der Primärversorgung die Notwendigkeit einer Kapsulodese oder einer Bandplastik mittels Sehnentransplantaten bestehen. Nach entsprechender ligamentärer Rekonstruktion sollten die betroffenen Gelenkanteile in der Re- 

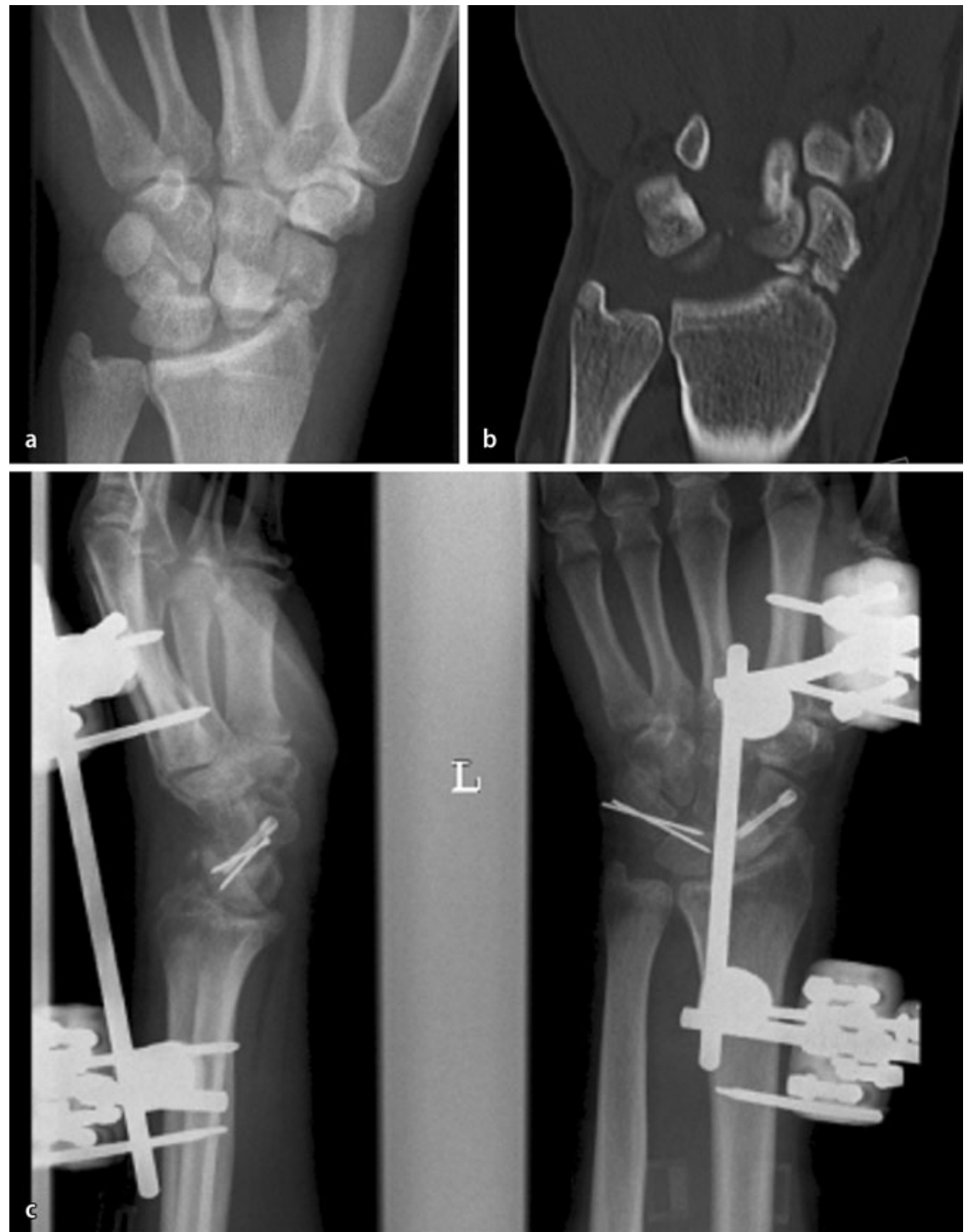

Abb. 1 \ Männlicher Patient, 25 Jahre, mit transskaphoidaler perilunärer Luxationsfraktur des linken Handgelenks mit Skaphoidfraktur und Ruptur des lunotriquetralen Bandes, a p.-a. Röntgenaufnahme mit Darstellung der Skaphoidfraktur sowie der karpalen Luxation, b mit Computertomographie genaue Beurteilung der knöchernen Trümmerzone im Bereich des distalen Skaphoidpols möglich, c Zustand nach offener Reposition, Herbert-Schrauben-Osteosynthese des Os scaphoideum, Naht des lunotriquetralen Bandes, temporärer Kirschner-Draht-Transfixation des lunotriquetralen Intervalls und Anlage eines Fixateur externe

gel mittels Kirschner-Drähten temporär transfixiert werden. Im Fall einer ausgeprägten Instabilität kann auch temporär ein Fixateur externe erforderlich werden

(• Abb. 1c).

Ausgedehnte und komplexe Destruktionen des Carpus erfordern häufig ein modifiziertes Vorgehen. So kann mitunter bereits im Rahmen der Erstversorgung bei einer kompletten Destruktion der distalen oder proximalen Handwurzelreihe die Exstirpation derselben als Rettungseingriff zum Erhalt einer Restbe- ation und eventuelle Sehnen- und Nervenverletzungen in die Beurteilung des Gesamtverletzungsausmaßes und die Entwicklung eines Therapieplanes einfließen. Eine transkarpale Amputationsverletzung erlaubt $u$. U. wegen der Notwendigkeit einer Minimierung der Ischämiezeit keine komplexen und zeitaufwendigen Osteosynthesen. Bei einem ausgedehnten Weichteiltrauma stellt deshalb der Fixateur externe eine sinnvolle Alternative dar. In der Regel sollte angestrebt werden, bereits im Rahmen der Erstversorgung eine definitive knöcherne und ligamentäre Rekonstruktion zu erzielen. Häufig lässt der Zustand der Weichteile eventuell erforderliche weitergehende Eingriffe im Bereich des Carpus erst zu einem sehr viel späteren Zeitpunkt wieder zu.

Knöcherne und ligamentäre Verletzungen im Rahmen einer schweren kombinierten Handverletzung sollten möglichst in einem spezialisierten Zentrum versorgt werden, da häufig bereits im Rahmen der Erstversorgung ausgedehnte mikrochirurgische Eingriffe bis hin zu Gefäßbypässen erforderlich sind und mitunter entweder bereits im Rahmen der Erstversorgung oder innerhalb weniger Tage nach dem Trauma die Rekonstruktion von Weichteildefekten mittels mikrochirurgischer Lappenplastiken notwendig werden kann $[9,18]$.

\section{Perioperatives Management und Nachbehandlung}

In der perioperativen Phase ist eine adäquate Schmerztherapie unerlässlich. Im Falle von Amputationsverletzungen sollte die betroffene Extremität ggf. gewärmt werden. Bei offenen Verletzungen ist häufig eine prophylaktische Antibiotikagabe indiziert.

Bereits in der frühpostoperativen Phase sollte mit der Physiotherapie begonnen werden, um den Abschwellungsprozess zu unterstützen und Einsteifungen im Bereich der Finger zu vermeiden. Dabei sollte das physiotherapeutische Prozedere eng mit dem Operateur abgestimmt werden [3]. Dies ist insbesondere bei zusätzlich bestehenden Nerven- und Sehnenverletzungen von großer Relevanz.

Die Immobilisierung des Handgelenks erfolgt in Abhängigkeit des Verletzungs- 


\section{Die schwere kombinierte Handverletzung}
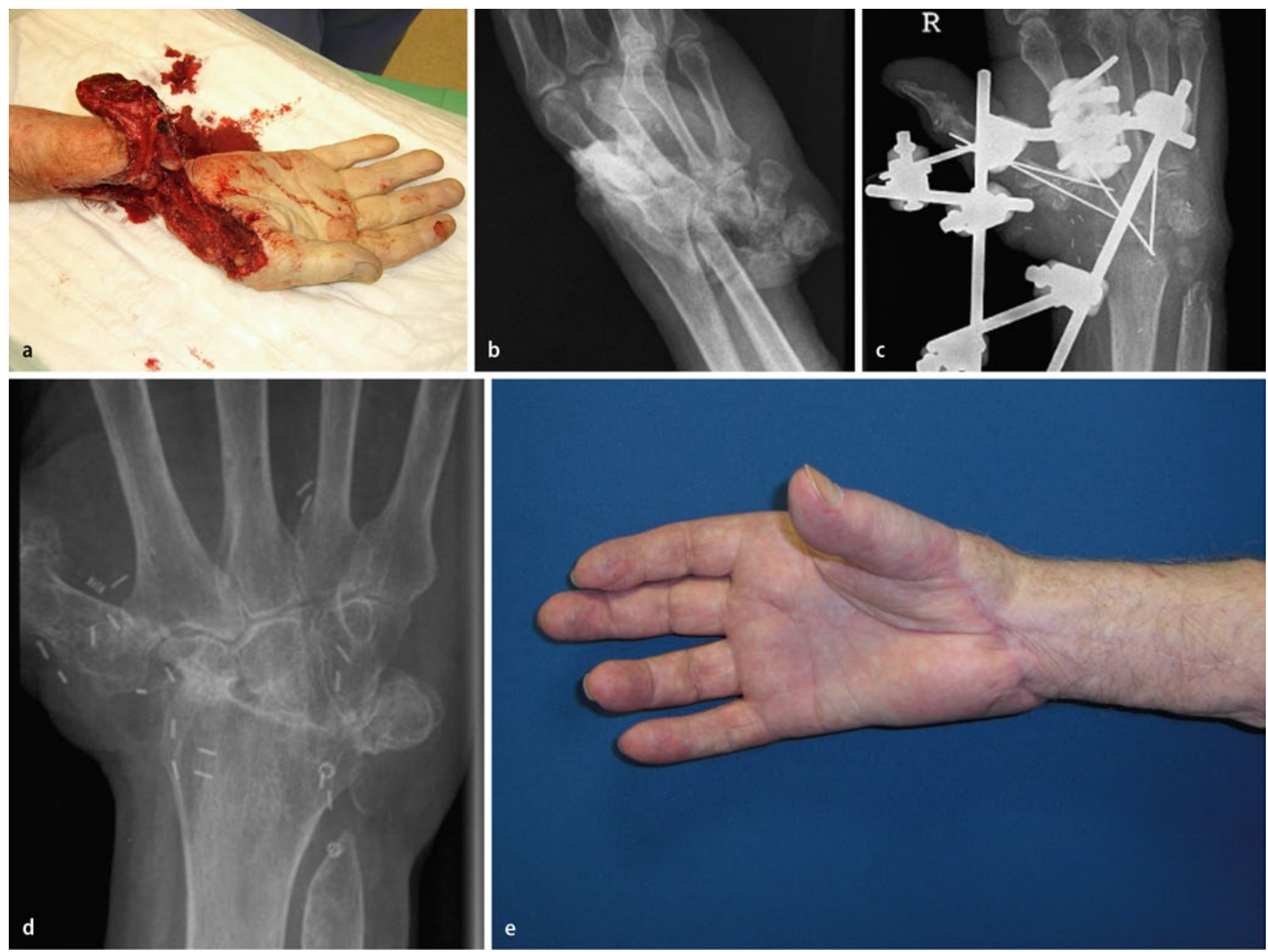

Abb. $2 \Delta$ Männlicher Patient, 76 Jahre, a,b subtotale transkarpale Handamputation mit kompletter Destruktion des Radiokarpalgelenks, der proximalen und teilweise der distalen Handwurzelreihe sowie des Ulnaköpfchens, c Zustand nach Replantation und modifizierter kompletter Handgelenkarthrodese mit Kirschner-Drähten und Fixateur externe sowie Resektion des Caput ulnae, d,e komplette knöcherne Konsolidierung und Abheilung der Weichteile nach 12 Monaten

ausmaßes und der im Bereich des Carpus durchgeführten Rekonstruktionsmaßnahmen. Während isolierte Skaphoidfrakturen bei übungsstabiler Versorgung bereits direkt postoperativ einer Übungsbehandlung zugeführt werden können, erfordern modifizierte Arthrodesen im Bereich des Carpus nach transkarpalen Amputationsverletzungen gelegentlich eine Immobilisierung über mehrere Monate. Die Bedeutung der physiound ergotherapeutischen Nachbehandlung bei knöchernen und ligamentären karpalen Verletzungen kann nicht überschätzt werden [22]. Schwere kombinierte Handverletzungen müssen häufig zur Erzielung eines optimalen Rehabilitationsergebnisses einer stationären Maßnahme [z. B. komplexe stationäre Rehabilitation (KSR), berufsgenossenschaftliche statio- näre Weiterbehandlung (BGSW)] oder einer erweiterten ambulanten Physiotherapie (EAP) zugeführt werden.

Wegen des hohen Risikos einer dauerhaften Berufsunfähigkeit aufgrund von bleibenden funktionellen Einschränkungen ist frühzeitig ein Berufshelfer hinzuzuziehen. Insbesondere bei jungen Patienten sollte ggf. frühzeitig unter Berücksichtigung der zu erwartenden funktionellen Reserven der verletzten Extremität eine Beratung bezüglich Umschulungsmaßnahmen erfolgen [8].

\section{Sekundäreingriffe}

Nach Abschluss der chirurgischen Primärtherapie kann sich im Verlauf die Notwendigkeit von weiteren Eingriffen im Bereich des Carpus ergeben. Ur- sächlich hierfür können entweder bei Zustand nach ligamentären Verletzungen sekundäre Gefügestörungen aufgrund von ligamentärer Instabilität sein oder auch die Entwicklung von sekundären Arthrosen. Des Weiteren können Störungen der knöchernen Durchbauung im Sinne von Pseudarthrosen weitergehende operative Eingriffe erforderlich machen.

Ziel aller sekundären operativen Maßnahmen nach knöchernen und ligamentären Verletzungen sollte immer der Erhalt einer schmerzfreien Restbeweglichkeit im Bereich des Handgelenks mit guter Kraftentfaltung sein.

Die häufigste karpale Gefügestörung nach ligamentären Verletzungen ist die DISI-Deformität (DISI: „dorsal intercalated segment instability"), bei der es zur Dorsalverkippung des Os lunatum mit 
gleichzeitiger Palmarverkippung des Os scaphoideum kommt. Ursächlich hierfür ist in der Regel eine Instabilität im Bereich des skaphulonären Bandes. In Abhängigkeit vom Ausmaß der Instabilität sowie der weiteren betroffenen Strukturen können Bandplastiken, Kapsulodesen oder auch Teilversteifungen, wie die Versteifung des STT-Gelenks (Skaphoid-Trapezium-Trapezoideum-Gelenk), indiziert sein.

Posttraumatische Arthrosen im Bereich des Medio- und Radiokarpalgelenks führen oft zu schmerzhaften Bewegungseinschränkungen und Kraftverlust in der betroffenen Hand. In Abhängigkeit von Lokalisation und Ausmaß des Befundes kann u. U. auch durch Teilversteifungen, wie eine mediokarpale Teilarthrodese nach Skaphoidektomie, oder eine Resektion der körpernahen Handwurzelreihe („proximal row carpectomy“) eine gute Restbeweglichkeit im Handgelenk bei suffizienter Kraftentfaltung erzielt werden [5, 17]. Als zusätzliche, palliative Maßnahme bewährte sich die partielle oder komplette Denervierung des Handgelenks [23].

Sowohl im Fall sekundärer Bandinstabilitäten als auch bei posttraumatischen Arthrosen kann die diagnostische Handgelenkarthroskopie wertvolle Informationen im Hinblick auf die Planung des weiteren therapeutischen Vorgehens geben.

Pseudarthrosen im Bereich der Carpalia, z. B. die Pseudarthrose des Os scaphoideum, erfordern in aller Regel eine operative Revision mit Pseudarthrosenresektion und autologer Knochentransplantation mit Revisionsosteosynthese. Unter Umständen kann bei therapierefraktären Skaphoidpseudarthrosen bei Durchblutungsstörungen im proximalen Fragment auch ein vaskularisierter Knochentransfer erforderlich werden [2]. Inadäquat versorgte Pseudarthrosen im Bereich der Carpalia können ebenfalls zur karpalen Gefügestörung und zum Auftreten einer sekundären Handgelenkarthrose führen. Im Fall von ausgeprägten sekundären Handgelenkarthrosen bzw. einer fortgeschrittenen Gelenkdestruktion erlaubt die Versteifung des Handgelenks in der Regel eine deutliche Beschwerdebesserung und Steigerung der Gebrauchsfähigkeit der betroffenen Extremität. Die Komplettarthrodese im Bereich des Handgelenks wird meist mittels Plattenosteosynthese und spongiösen oder kortikospongiösen Knochentransplantaten durchgeführt [10].

\section{Fazit für die Praxis}

Die chirurgische Therapie von knöchernen und ligamentären Verletzungen des Handgelenks im Kontext der schweren kombinierten Handverletzung stellt eine chirurgische Herausforderung dar. Dabei sind $u$. U. bereits im Rahmen der Primärversorgung neben der knöchernen und ligamentären Rekonstruktion v. a. bei Amputationsverletzungen mikrochirurgische Eingriffe, wie Nerven- und Gefäßnähte sowie u. U. komplexe Weichteileingriffe bis hin zu mikrochirurgischen freien Gewebetransfers erforderlich. Die Versorgung derartiger Verletzungen sollte in der Regel in entsprechend spezialisierten Zentren erfolgen. Die Rehabilitationsphase beginnt bei derartig komplexen Verletzungen direkt postoperativ, sodass entsprechende physio- und ergotherapeutische Kapazitäten bereits im Rahmen der primären stationären Therapie zwingend vorgehalten werden müssen. Eventuell sollte sich der akut stationären Versorgung eine stationäre Rehabilitationsbehandlung in spezialisierten Einrichtungen anschließen.

\section{Korrespondenzadresse}

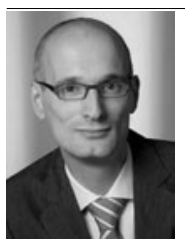

\section{PD Dr. U. Kneser}

Plastisch- und

Handchirurgische Klinik, Universitätsklinikum Erlangen, Krankenhausstraße 12, 91054 Erlangen Ulrich.Kneser@uk-erlangen.de

Interessenkonflikt. Der korrespondierende Autor gibt an, dass kein Interessenkonflikt besteht.

The supplement containing this article is not sponsored by industry.

\section{Literatur}

1. Berger RA (1996) The anatomy and basic biomechanics of the wrist joint. J Hand Ther 9:84-93

2. Burger KH, Gaggl AJ, Kukutschki W et al (2009) Free microvascular transfer of segmental corticocancellous femur for treatment of avascular scaphoid necrosis. Handchir Mikrochir Plast Chir 41:44-51
3. Chan SW, LaStayo P (2003) Hand therapy management following mutilating hand injuries. Hand Clin 19:133-148, vii

4. Cooney WP, Bussey R, Dobyns JH et al (1987) Difficult wrist fractures. Perilunate fracture-dislocations of the wrist. Clin Orthop Relat Res 214:136-147

5. Dacho AK, Baumeister S, Germann G et al (2008) Comparison of proximal row carpectomy and midcarpal arthrodesis for the treatment of scaphoid nonunion advanced collapse (SNAC-wrist) and scapholunate advanced collapse (SLAC-wrist) in stage II. J Plast Reconstr Aesthet Surg 61:1210-1218

6. Del Pinal F (2007) Severe mutilating injuries to the hand: guidelines for organizing the chaos. J Plast Reconstr Aesthet Surg 60:816-827

7. Germann G, Karle B, Bruner S et al (2000) Treatment strategy in complex hand injuries. Unfallchirurg 103:342-347

8. Hamacher E (1990) Rehabilitation measures and the expenditures of professional organizations for the treatment and compensation of patients with hand injuries. Handchir Mikrochir Plast Chir 22:227-231

9. Horch RE, Stark GB (1999) The rectus abdominis free flap as an emergency procedure in extensive upper extremity soft-tissue defects. Plast Reconstr Surg 103:1421-1427

10. Kalb K, Prommersberger KJ (2009) Total wrist fusion using the $\mathrm{AO}$ wrist fusion plate. Oper Orthop Traumatol 21:498-509

11. Kijima Y, Viegas SF (2009) Wrist anatomy and biomechanics. J Hand Surg [Am] 34:1555-1563

12. Krimmer H, Schmitt R, Herbert $T$ (2000) Scaphoid fractures. Diagnosis, classification and therapy. Unfallchirurg 103:812-819

13. Kuo CE, Wolfe SW (2008) Scapholunate instability: current concepts in diagnosis and management. J Hand Surg [Am] 33:998-1013

14. Lichtman DM, Martin RA (1988) Introduction to the carpal instabilities. In: Lichtman DM (Hrsg) The wrist and its disorders. Saunders, Philadelphia, pp 244-250

15. Mayfield JK, Johnson RP, Kilcoyne RF (1976) The ligaments of the human wrist and their functional significance. Anat Rec 186:417-428

16. Mayfield JK, Johnson RP, Kilcoyne RK (1980) Carpal dislocations: pathomechanics and progressive perilunar instability. J Hand Surg [Am] 5:226-241

17. Mulford JS, Ceulemans LJ, Nam D et al (2009) Proximal row carpectomy vs four corner fusion for scapholunate (Slac) or scaphoid nonunion advanced collapse (Snac) wrists: a systematic review of outcomes. J Hand Surg Eur 34:256-263

18. Neumeister MW, Brown RE (2003) Mutilating hand injuries: principles and management. Hand Clin 19:1-15, v

19. Schmidt HM, Lanz U (2003) Chirurgische Anatomie der Hand. Thieme, Stuttgart New York

20. Slutsky D (2009) Principles and practice of wrist surgery. Saunders Elsevier, Philadelphia

21. Taleisnik J (1976) The ligaments of the wrist. J Hand Surg [Am] 1:110-118

22. Vollinger C, Partecke BD (2003) Nachbehandlung nach distalen Unterarmfrakturen und Handwurzelverletzungen. Orthopade 32:380-385

23. Wilhelm A (1974) Denervation of the wrist. Z Unfallmed Berufskrankh 67:113-119

24. Wolfe SW, Hotchkiss RN, Pederson WC et al (2010) Green's operative hand surgery. Elsevier Churchill Livingstone, Philadelphia

25. Woo SH, Lee YK, Lee HH et al (2009) Hand replantation with proximal row carpectomy. Hand (NY) 4:55-61 